\title{
CONTRACTIVE DETERMINANTAL REPRESENTATIONS OF STABLE POLYNOMIALS ON A MATRIX POLYBALL
}

\author{
ANATOLII GRINSHPAN, DMITRY S. KALIUZHNYI-VERBOVETSKYI, VICTOR VINNIKOV, \\ AND HUGO J. WOERDEMAN
}

\begin{abstract}
We show that an irreducible polynomial $p$ with no zeros on the closure of a matrix unit polyball, a.k.a. a cartesian product of Cartan domains of type I, and such that $p(0)=1$, admits a strictly contractive determinantal representation, i.e., $p=\operatorname{det}\left(I-K Z_{n}\right)$, where $n=\left(n_{1}, \ldots, n_{k}\right)$ is a $k$-tuple of nonnegative integers, $Z_{n}=\bigoplus_{r=1}^{k}\left(Z^{(r)} \otimes I_{n_{r}}\right), Z^{(r)}=$ $\left[z_{i j}^{(r)}\right]$ are complex matrices, $p$ is a polynomial in the matrix entries $z_{i j}^{(r)}$, and $K$ is a strictly contractive matrix. This result is obtained via a noncommutative lifting and a theorem on the singularities of minimal noncommutative structured system realizations.
\end{abstract}

\section{INTRODUCTION}

Polynomial stability arises naturally in various problems of Analysis and its applications such as Electrical Engineering and Control Theory [8, 26, 7, 19, 9, 14, 23, 22]. A polynomial $p \in \mathbb{C}\left[z_{1}, \ldots, z_{d}\right]$ is called stable with respect to a domain $\mathcal{D} \subseteq \mathbb{C}^{d}$, or just $\mathcal{D}$-stable, if it has no zeros in $\mathcal{D}$, and strongly $\mathcal{D}$-stable if it has no zeros in the domain closure $\overline{\mathcal{D}}$. In the case where $d=1$ and $\mathcal{D}$ is the unit disk $\mathbb{D}=\{z \in \mathbb{C}:|z|<1\}$, and $p(0)=1$, one can write

$$
p=\left(1-a_{1} z\right) \cdots\left(1-a_{n} z\right)=\operatorname{det}(I-K z),
$$

where $a_{i}=1 / z_{i}, i=1, \ldots, n$, the zeros $z_{i}$ of $p$ are counted according to their multiplicities, $K=\operatorname{diag}\left[a_{1}, \ldots, a_{n}\right]$, and $n=\operatorname{deg} p$. It follows that the matrix $K$ is contractive (resp., strictly contractive), i.e., $\|K\| \leq 1$ (resp., $\|K\|<1$ ); here and throughout the paper, $\|\cdot\|$ is the operator $(2,2)$ norm.

In the case where $d=2$ and $\mathcal{D}$ is the unit bidisk $\mathbb{D}^{2}$, it is also true that a stable (resp., strongly stable) polynomial $p$ has a contractive (resp., strictly contractive) determinantal representation. It was shown in [10] (see also [20, 19, 21]) that every $\mathbb{D}^{2}$-stable (resp., strongly $\mathbb{D}^{2}$-stable) polynomial $p$, with $p(0)=1$, can be represented as

$$
p=\operatorname{det}\left(I-K Z_{n}\right),
$$

where $n=\left(n_{1}, n_{2}\right) \in \mathbb{Z}_{+}^{2}$ is the bi-degree of $p, Z_{n}=\operatorname{diag}\left[z_{1} I_{n_{1}}, z_{2} I_{n_{2}}{ }^{1}\right]$, and the matrix $K$ is contractive (resp., strictly contractive).

For a higher-dimensional polydisk case, $\mathcal{D}=\mathbb{D}^{d}, d>2$, it is in general not true that every stable (resp., strongly stable) polynomial $p$, with $p(0)=1$, has a determinantal representation

1991 Mathematics Subject Classification. 15A15, 32A10, 47N70, 14A22.

Key words and phrases. Contractive determinantal representation; stable polynomial; polyball; classical Cartan domain; contractive realization; structured noncommutative multidimensional system.

AG, DK-V, HW were partially supported by NSF grant DMS-0901628. DK-V and VV were partially supported by BSF grant 2010432.

${ }^{1}$ Here and in the rest of the paper we use a convention that a matrix block which involves $I_{n_{i}}$ is void in the case of $n_{i}$ equal to 0 . 
(1.1) where now $n=\left(n_{1}, \ldots, n_{d}\right) \in \mathbb{Z}_{+}^{d}$ is equal to the multi-degree of $p, \operatorname{deg} p, Z_{n}=$ $\operatorname{diag}\left[z_{1} I_{n_{1}}, \ldots, z_{d} I_{n_{d}}\right]$, and the matrix $K$ is contractive (resp., strictly contractive). Such a representation with $n \geq \operatorname{deg} p$ (in the sense that $n_{i} \geq \operatorname{deg}_{i} p, i=1, \ldots, d$, where $\operatorname{deg}_{i} p$ denotes the $i$-th partial degree of $p$ ) has been constructed for some special classes of stable polynomials in [12.

The existence of a representation (1.1) with a contractive (resp., strictly contractive) matrix $K$ provides a certificate for stability (resp., strong stability) of a polynomial $p$. Moreover, if merely a polynomial multiple of $p$ has such a representation, the stability (resp., strong stability) of $p$ is guaranteed. In a recent paper of the authors [11, the following result has been obtained. Let $\mathbf{P} \in \mathbb{C}^{\ell \times m}\left[z_{1}, \ldots, z_{d}\right]$ and $\mathbf{P}^{(r)} \in \mathbb{C}^{\ell_{r} \times m_{r}}\left[z_{1}, \ldots, z_{d}\right], r=1, \ldots, k$, be such that $\mathbf{P}=\bigoplus_{r=1}^{k} \mathbf{P}^{(r)}$, and let

$$
\mathcal{D}_{\mathbf{P}}=\left\{z \in \mathbb{C}^{d}:\|\mathbf{P}(z)\|<1\right\} .
$$

Under an appropriate Archimedean condition on $\mathbf{P}$, which in particular implies the boundedness of the domain $\mathcal{D}_{\mathbf{P}}$, for every strongly $\mathcal{D}_{\mathbf{P}}$-stable polynomial $p \in \mathbb{C}\left[z_{1}, \ldots, z_{d}\right]$ there exists a polynomial $q \in \mathbb{C}\left[z_{1}, \ldots, z_{d}\right]$ such that $p q$ has a determinantal representation

$$
p q=\operatorname{det}\left(I-K \mathbf{P}_{n}\right),
$$

where $n=\left(n_{1}, \ldots, n_{k}\right) \in \mathbb{Z}_{+}^{k}, \mathbf{P}_{n}=\bigoplus_{r=1}^{k}\left(\mathbf{P}^{(r)} \otimes I_{n_{r}}\right)$, and the matrix $K$ is strictly contractive. We note that special cases of the domain $\mathcal{D}_{\mathbf{P}}$ as above include the unit polydisk and the classical Cartan domains of type I, II, and III, as well as the Cartesian products of such domains.

In this paper, we construct strictly contractive determinantal representations for strongly stable polynomials on a Cartesian product of Cartan's domains of type I, i.e., on a matrix unit polyball,

$$
\begin{aligned}
\mathbb{B}^{\ell_{1} \times m_{1}} & \times \cdots \times \mathbb{B}^{\ell_{k} \times m_{k}} \\
& =\left\{Z=\left(Z^{(1)}, \ldots, Z^{(k)}\right) \in \mathbb{C}^{\ell_{1} \times m_{1}} \times \cdots \times \mathbb{C}^{\ell_{k} \times m_{k}}:\left\|Z^{(r)}\right\|<1, r=1, \ldots, k\right\} .
\end{aligned}
$$

In other words, in the case where $\mathcal{D}_{\mathbf{P}}$ is a unit matrix polyball, i.e., where $\mathbf{P}^{(r)}=Z^{(r)}$, $r=1, \ldots, k$, no additional polynomial factor $q$ is needed to construct a strictly contractive determinantal representation (1.3), and we have

$$
p=\operatorname{det}\left(I-K Z_{n}\right), \quad Z_{n}=\bigoplus_{r=1}^{k}\left(Z^{(r)} \otimes I_{n_{r}}\right)
$$

with some $n=\left(n_{1}, \ldots, n_{k}\right) \in \mathbb{Z}_{+}^{k}$ and some $\sum_{r=1}^{k} m_{r} n_{r} \times \sum_{r=1}^{k} \ell_{r} n_{r}$ matrix $K$ such that $\|K\|<1$. Notice that the unit polydisk $\mathbb{D}^{d}$ is a special case of a unit matrix polyball where $k=d$, and $\ell_{r}=m_{r}=1$ for $r=1, \ldots, k$.

The proof of our main theorem has two components: realization formulas for multivariable rational functions and related techniques from multidimensional system theory, and results from a theory of noncommutative rational functions.

The first component was a key for constructing determinantal representations in [20, 21, 19, 12, 10, 11]. We recall (see [3, Proposition 11]) that every matrix-valued rational function that is regular and contractive on the open unit disk $\mathbb{D}$ can be realized as

$$
F=D+z C(I-z A)^{-1} B
$$


with a contractive colligation matrix $\left[\begin{array}{cc}A & B \\ C & D\end{array}\right]$. In several variables, the celebrated result of Agler [1] gives the existence of a realization of the form

$$
F(z)=D+C Z_{\mathcal{X}}\left(I-A Z_{\mathcal{X}}\right)^{-1} B, \quad Z_{\mathcal{X}}=\bigoplus_{i=1}^{d} z_{i} I_{\mathcal{X}_{i}},
$$

where $z=\left(z_{1}, \ldots, z_{d}\right) \in \mathbb{D}^{d}$ and the colligation $\left[\begin{array}{cc}A & B \\ C & D\end{array}\right]$ is a Hilbert-space unitary operator (with $A$ acting on the orthogonal direct sum of Hilbert spaces $\mathcal{X}_{1}, \ldots, \mathcal{X}_{d}$ ), for $F$ an operatorvalued function holomorphic on the unit polydisk $\mathbb{D}^{d}$ whose Agler norm

$$
\|F\|_{\mathcal{A}}=\sup _{T \in \mathcal{T}}\|F(T)\|
$$

is at most 1 . Here $\mathcal{T}$ is the set of $d$-tuples $T=\left(T_{1}, \ldots, T_{d}\right)$ of commuting strict contractions on a Hilbert space. Such functions constitute the Schur-Agler class.

Agler's result was generalized to polynomially defined domains in 2, 4]. Given $\mathbf{P} \in$ $\mathbb{C}^{\ell \times m}\left[z_{1}, \ldots, z_{d}\right]$, let $\mathcal{D}_{\mathbf{P}}$ be as in (1.2) (here we can assume that $k=1$ and $\mathbf{P}=\mathbf{P}_{1}$ ) and let $\mathcal{T}_{\mathbf{P}}$ be the set of $d$-tuples $T$ of commuting bounded operators on a Hilbert space satisfying $\|\mathbf{P}(T)\|<1$. (The case of unit polydisk $\mathbb{D}^{d}$ corresponds to $\mathbf{P}=\operatorname{diag}\left[z_{1}, \ldots, z_{d}\right]$ and $\mathcal{T}_{\mathbf{P}}=\mathcal{T}$.) For $T \in \mathcal{T}_{\mathbf{P}}$, the Taylor joint spectrum $\sigma(T)$ [24] lies in $\mathcal{D}_{\mathbf{P}}$ (see [2, Lemma 1]), and therefore for an operator-valued function $F$ holomorphic on $\mathcal{D}_{\mathbf{P}}$ one defines $F(T)$ by means of Taylor's functional calculus [25] and

$$
\|F\|_{\mathcal{A}, \mathbf{P}}=\sup _{T \in \mathcal{T}_{\mathbf{P}}}\|F(T)\|
$$

We say that $F$ belongs to the operator-valued Schur-Agler class associated with $\mathbf{P}$, denoted by $\mathcal{S} \mathcal{A}_{\mathbf{P}}(\mathcal{U}, \mathcal{Y})$, if $F$ is holomorphic on $\mathcal{D}_{\mathbf{P}}$, takes values in the space $\mathcal{L}(\mathcal{U}, \mathcal{Y})$ of bounded linear operators from a Hilbert space $\mathcal{U}$ to a Hilbert space $\mathcal{Y}$, and $\|F\|_{\mathcal{A}, \mathbf{P}} \leq 1$.

The generalization of Agler's theorem mentioned above that has appeared first in [2] for the scalar-valued case and extended in [4] to the operator-valued case, says that a function $F$ belongs to the Schur-Agler class $\mathcal{S} \mathcal{A}_{\mathbf{P}}(\mathcal{U}, \mathcal{Y})$ if and only if there exist a Hilbert space $\mathcal{X}$ and a unitary colligation

$$
\left[\begin{array}{ll}
A & B \\
C & D
\end{array}\right]:\left(\mathbb{C}^{m} \otimes \mathcal{X}\right) \oplus \mathcal{U} \rightarrow\left(\mathbb{C}^{\ell} \otimes \mathcal{X}\right) \oplus \mathcal{Y}
$$

such that

$$
F(z)=D+C\left(\mathbf{P}(z) \otimes I_{\mathcal{X}}\right)\left(I-A\left(\mathbf{P}(z) \otimes I_{\mathcal{X}}\right)\right)^{-1} B
$$

If the Hilbert spaces $\mathcal{U}$ and $\mathcal{Y}$ are finite-dimensional, $F$ can be treated as a matrix-valued function (relative to a pair of orthonormal bases for $\mathcal{U}$ and $\mathcal{Y}$ ). It is natural to ask whether every rational $\alpha \times \beta$ matrix-valued function in the Schur-Agler class $\mathcal{S A}_{\mathbf{P}}\left(\mathbb{C}^{\beta}\right.$, $\left.\mathbb{C}^{\alpha}\right)$ has a realization (1.6) with a contractive colligation matrix $\left[\begin{array}{cc}A & B \\ C & D\end{array}\right]$. This question is open for $d>1$, except for the following two cases. The first case is when $F$ is an inner (i.e., regular on $\mathbb{D}^{d}$ and taking unitary boundary values a.e. on the unit torus $\mathbb{T}^{d}=\left\{z=\left(z_{1}, \ldots, z_{d}\right) \in \mathbb{C}^{d}:\left|z_{i}\right|=\right.$ $1, i=1, \ldots, d\})$ matrix-valued Schur-Agler function on $\mathbb{D}^{d}$. In this case, the colligation matrix for the realization (1.6) can be chosen unitary; see [18] for the scalar-valued case, and [6, Theorem 2.1] for the matrix-valued generalization. We notice here that not every rational inner function is Schur-Agler; see [12, Example 5.1] for a counterexample. In the second case, one assumes that $\mathbf{P}=\bigoplus_{r=1}^{k} \mathbf{P}^{(r)}$ satisfies a certain matrix-valued Archimedean 
condition and $F$ is regular on the closed domain $\overline{\mathcal{D}}_{\mathbf{P}}$ and satisfies $\|F\|_{\mathcal{A}, \mathbf{P}}<1$. Then there exists a contractive finite-dimensional realization of $F$ in the form

$$
F=D+C \mathbf{P}_{n}\left(I-A \mathbf{P}_{n}\right)^{-1} B, \quad \mathbf{P}_{n}=\bigoplus_{r=1}^{k}\left(\mathbf{P}^{(r)} \otimes I_{n_{r}}\right),
$$

with some $n=\left(n_{1}, \ldots, n_{k}\right) \in \mathbb{Z}_{+}^{k}[11]$.

The second component in the proof of our main result, a theory of noncommutative rational functions, is briefly summarized in Section 2. Then a version of a theorem from [17] on the singularities of a noncommutative rational matrix-valued function in terms of its minimal realization, where the realization is in the form of a structured noncommutative multidimensional system, i.e., the one that is associated with a unit polyball (1.4), is proved (see [5] for details on structured noncommutative multidimensional systems). As a corollary, an analogous theorem on the singularities of a commutative matrix-valued rational function is obtained via a noncommutative lifting.

In Section 3, our main theorem is proved, which establishes the existence of a strictly contractive determinantal representation for every irreducible strongly stable polynomial on a matrix polyball. As a corollary, in the case of the unit polydisk $\mathbb{D}^{d}$, we obtain that every strongly stable polynomial $p$ is an eventual Agler denominator, i.e., is the denominator of a rational inner function of the Schur-Agler class.

\section{Singularities of NONCOMMUtATIVE RATiONAL FUnCTIONS AND Minimal STRUCTURED NONCOMMUTATIVE MULTIDIMENSIONAL SYSTEMS}

We first give some necessary background on matrix-valued noncommutative rational functions; see [17, 16] for more details, and we also refer to [15] for a general theory of free noncommutative functions.

A matrix-valued noncommutative rational expression $R$ over a field $\mathbb{K}$ is any expression obtained from noncommuting indeterminates $z_{1}, \ldots, z_{d}$, and a constant $1 \in \mathbb{K}$ by successive elementary operations: addition, multiplication, and inversion, forming (block) matrices, and also matrix addition, multiplication, and inversion. E.g., an $\alpha \times \beta$ matrix-valued noncommutative polynomial

$$
R=\sum_{w \in \mathcal{G}_{d}:|w| \leq L} R_{w} z^{w}
$$

is a matrix-valued noncommutative rational expression defined without using inversions. Here $\mathcal{G}_{d}$ is the free monoid on $d$ generators (letters) $g_{1}, \ldots, g_{d}$, the coefficients $R_{w}$ are $\alpha \times \beta$ matrices over $\mathbb{K}$, and for an element $w=g_{i_{1}} \cdots g_{i_{N}} \in \mathcal{G}_{d}$ (a word in the alphabet $g_{1}, \ldots, g_{d}$ ) we set $z^{w}=z_{i_{1}} \cdots z_{i_{N}}$ and $z^{\emptyset}=1$, where $\emptyset$ is the unit element of $\mathcal{G}_{d}$ (the empty word), and $|w|=N$ is the length of the word $w$, in particular $|\emptyset|=0$.

For a $d$-tuple $Z=\left(Z_{1}, \ldots, Z_{d}\right)$ of $s \times s$ matrices over $\mathbb{K}$, one can evaluate

$$
R(Z)=R_{s}(Z)=\sum_{w \in \mathcal{G}_{d}} R_{w} \otimes Z^{w}
$$

where $Z^{w}=Z_{i_{1}} \cdots Z_{i_{k}}$ and $Z^{\emptyset}=I_{s}$. Similarly, one can evaluate $R$ on a $d$-tuple $X=$ $\left(X_{1}, \ldots, X_{d}\right)$ of generic $s \times s$ matrices, i.e., on a $d$-tuple of matrices over commuting indeterminates $\left(X_{r}\right)_{i j}, r=1, \ldots, d, i, j=1, \ldots, s$. We then define evaluations $R(Z)=R_{s}(Z)$ and $R(X)=R_{s}(X)$ whenever all the formal matrix inversions in the expression $R$ can be 
replaced by matrix inversions for matrices over $\mathbb{K}$ (resp., for generic matrices); this defines the domain of regularity of $R, \operatorname{dom}_{s} R$, and the extended domain of regularity of $R, \operatorname{edom}_{s} R$, inside the set of $d$-tuples of $s \times s$ matrices over $\mathbb{K}$ ( $d$-tuples of generic matrices). Then one defines $\operatorname{dom} R=\coprod_{s=1}^{\infty} \operatorname{dom}_{s} R$ and edom $R=\coprod_{s=1}^{\infty} \operatorname{edom}_{s} R$. One has

$$
\operatorname{edom}_{s} R \supseteq \operatorname{dom}_{s} R, \quad \operatorname{edom} R \supseteq \operatorname{dom} R .
$$

Two $\alpha \times \beta$ matrix-valued noncommutative rational expressions $R_{1}$ and $R_{2}$ are called equivalent if $\operatorname{dom} R_{1} \cap \operatorname{dom} R_{2} \neq \emptyset$ and $R_{1}(Z)=R_{2}(Z)$ for every $Z \in \operatorname{dom} R_{1} \cap \operatorname{dom} R_{2}$. An equivalence class of $\alpha \times \beta$ matrix-valued noncommutative rational expressions is called an $\alpha \times \beta$ matrix-valued noncommutative rational function. We write $R \in \mathfrak{R}$ if a matrix-valued noncommutative rational function $\mathfrak{R}$ as an equivalence class of matrix-valued noncommutative rational expressions contains $R$. We define

$$
\operatorname{dom}_{s} \mathfrak{R}=\bigcap_{R \in \mathfrak{R}} \operatorname{dom}_{s} R, \quad \operatorname{dom} \mathfrak{R}=\coprod_{s=1}^{\infty} \operatorname{dom}_{s} \mathfrak{R} .
$$

Next, we observe that if $R_{1}$ and $R_{2}$ are equivalent, then their evaluations on generic matrices give rise to the same $\alpha \times \beta$ matrix-valued commutative rational function, so $\operatorname{edom}_{s} R_{1}=$ $\operatorname{edom}_{s} R_{2}$ for every $s$. Therefore, we can define

$$
\operatorname{edom}_{s} \mathfrak{R}=\operatorname{edom}_{s} R, \quad \operatorname{edom} \mathfrak{R}=\operatorname{edom} R
$$

for any $R \in \mathfrak{R}$. Clearly, we have

$$
\operatorname{edom}_{s} \mathfrak{R} \supseteq \operatorname{dom}_{s} \mathfrak{R}, \quad \operatorname{edom} \mathfrak{R} \supseteq \operatorname{dom} \mathfrak{R} .
$$

In [17, the left and right backward shift operators $\mathcal{L}_{j}$ and $\mathcal{R}_{j}, j=1, \ldots, d$, were defined for matrix-valued noncommutative rational expressions. It was shown that if $R_{1}$ and $R_{2}$ are equivalent, then so are $\mathcal{L}_{j}\left(R_{1}\right)$ and $\mathcal{L}_{j}\left(R_{2}\right)$ (resp., $\mathcal{R}_{j}\left(R_{1}\right)$ and $\mathcal{R}_{j}\left(R_{2}\right)$ ). Therefore, these definitions can be extended to matrix-valued noncommutative rational functions. One defines

however we have

$$
\operatorname{dom} \mathcal{L}_{j}(R)=\operatorname{dom} \mathcal{R}_{j}(R)=\operatorname{dom} R
$$

and therefore

$$
\operatorname{edom} \mathcal{L}_{j}(R) \supseteq \operatorname{edom} R, \quad \operatorname{edom} \mathcal{R}_{j}(R) \supseteq \operatorname{edom} R,
$$

$$
\operatorname{edom} \mathcal{L}_{j}(\mathfrak{R}) \supseteq \operatorname{edom} \mathfrak{R}, \quad \operatorname{edom} \mathcal{R}_{j}(\mathfrak{R}) \supseteq \operatorname{edom} \mathfrak{R} .
$$

We will not need the general definitions of the left and right backward shifts here. It suffices for us to use the fact that every matrix-valued noncommutative rational function $\mathfrak{R}$ which is regular at 0 , i.e., such that $0 \in \operatorname{dom}_{1} \mathfrak{R}$, has a formal power series expansion

$$
\mathfrak{R} \sim \sum_{w \in \mathcal{G}_{d}} \mathfrak{R}_{w} z^{w}
$$

whose evaluation on $s \times s$ matrices is convergent in some neighborhood of zero for each $s$, and that

$$
\mathcal{L}_{j} \mathfrak{R} \sim \sum_{w \in \mathcal{G}_{d}} \mathfrak{R}_{g_{j} w} z^{w}, \quad \mathcal{R}_{j} \mathfrak{R} \sim \sum_{w \in \mathcal{G}_{d}} \mathfrak{R}_{w g_{j}} z^{w} .
$$

The following theorem is a structured-system analogue of [17, Theorem 3.1]; for details on structured noncommutative multidimensional systems, see [5]. We note that we are not using here a bipartite-graph formalism adopted in [5] for system evolutions and, as a consequence, 
for the definitions of controllability and observability. Instead, we use more direct blockmatrix notations. The diligent reader can easily find the one-to-one correspondence between the two formalisms.

Theorem 2.1. Let $\mathfrak{R}$ be an $\alpha \times \beta$ matrix-valued noncommutative rational function over $a$ field $\mathbb{K}$ represented by the expression

$$
R=D+C z_{n}\left(I-A z_{n}\right)^{-1} B
$$

where $n=\left(n_{1}, \ldots, n_{k}\right) \in \mathbb{Z}_{+}^{k}, z_{n}=\bigoplus_{r=1}^{k}\left(z^{(r)} \otimes I_{n_{r}}\right), z^{(r)}=\left[z_{i j}^{(r)}\right]$ is a $\ell_{r} \times m_{r}$ matrix whose entries $z_{i j}^{(r)}$ are noncommuting indeterminates, $\left[\begin{array}{cc}A & B \\ C\end{array}\right] \in \mathbb{K}^{\left(\sum_{r=1}^{k} m_{r} n_{r}+\alpha\right) \times\left(\sum_{r=1}^{k} \ell_{r} n_{r}+\beta\right)}$ is a block matrix whose blocks $A, B, C$ have further block decomposition \&: $A=\left[A^{\left(r r^{\prime}\right)}\right]_{r, r^{\prime}=1, \ldots, k}$ with blocks $A^{\left(r r^{\prime}\right)} \in \mathbb{K}^{m_{r} \times \ell_{r^{\prime}}} \otimes \mathbb{K}^{n_{r} \times n_{r^{\prime}}} \cong\left(\mathbb{K}^{n_{r} \times n_{r^{\prime}}}\right)^{m_{r} \times \ell_{r^{\prime}}}$, so that for $j=1, \ldots, m_{r}, i=$ $1, \ldots, \ell_{r^{\prime}}$ one has $A_{j i}^{\left(r r^{\prime}\right)} \in \mathbb{K}^{n_{r} \times n_{r^{\prime}}} ; B=\operatorname{col}_{r=1, \ldots, k}\left[B^{(r)}\right]$ with blocks $B^{(r)} \in \mathbb{K}^{m_{r} \times 1} \otimes \mathbb{K}^{n_{r} \times \beta} \cong$ $\left(\mathbb{K}^{n_{r} \times \beta}\right)^{m_{r} \times 1}$, so that for $j=1, \ldots, m_{r}$ one has $B_{j}^{(r)} \in \mathbb{K}^{n_{r} \times \beta} ; C=\operatorname{row}_{i=1, \ldots, k}\left[C^{(r)}\right]$ with blocks $C^{(r)} \in \mathbb{K}^{1 \times \ell_{r}} \otimes \mathbb{K}^{\alpha \times n_{r}} \cong\left(\mathbb{K}^{\alpha \times n_{r}}\right)^{1 \times \ell_{r}}$, so that for $i=1, \ldots, \ell_{r}$ one has $C_{i}^{(r)} \in \mathbb{K}^{\alpha \times n_{r}}$; and $D \in \mathbb{K}^{\alpha \times \beta}$. Assume that the realization $R$ of $\mathfrak{R}$ as in (2.1) is minimal, or equivalently, controllable, i.e., for each $r_{0} \in\{1, \ldots, k\}$ and $j_{0} \in\left\{1, \ldots, m_{r_{0}}\right\}$ one has

$$
\sum_{N \in \mathbb{N}, \gamma \in\{1, \ldots, N\}, i_{\gamma} \in\left\{1, \ldots, \ell_{r_{\gamma}}\right\}, j_{\gamma} \in\left\{1, \ldots, m_{r_{\gamma}}\right\}} \operatorname{range}\left(A_{j_{0} i_{1}}^{\left(r_{0} r_{1}\right)} \cdots A_{j_{N-1} i_{N}}^{\left(r_{N-1} r_{N}\right)} B_{j_{N}}^{\left(r_{N}\right)}\right)=\mathbb{K}^{n_{r_{0}}}
$$

and observable, i.e., for each $r_{0} \in\{1, \ldots, k\}$ and $i_{0} \in\left\{1, \ldots, \ell_{r_{0}}\right\}$ one has

$$
\bigcap_{N \in \mathbb{N}, \gamma \in\{1, \ldots, N\}, i_{\gamma} \in\left\{1, \ldots, \ell_{r_{\gamma}}\right\}, j_{\gamma} \in\left\{1, \ldots, m_{r_{\gamma}}\right\}} \operatorname{ker}\left(C_{i_{N}}^{\left(r_{N}\right)} A_{j_{N} i_{N-1}}^{\left(r_{N} r_{N-1}\right)} \cdots A_{j_{1} i_{0}}^{\left(r_{1} r_{0}\right)}\right)=\{0\} .
$$

Then

$$
\begin{aligned}
\operatorname{edom} \mathfrak{R}=\operatorname{dom} \mathfrak{R}=\coprod_{s=1}^{\infty}\{ & Z=\left(Z^{(1)}, \ldots, Z^{(k)}\right) \in\left(\mathbb{K}^{s \times s}\right)^{\ell_{1} \times m_{1}} \times \cdots \times\left(\mathbb{K}^{s \times s}\right)^{\ell_{k} \times m_{k}} \\
& \left.\cong\left(\mathbb{K}^{\ell_{1} \times m_{1}} \times \cdots \times \mathbb{K}^{\ell_{k} \times m_{k}}\right) \otimes \mathbb{K}^{s \times s}: \operatorname{det}(I-A \odot Z) \neq 0\right\},
\end{aligned}
$$

where $A \odot Z \in \mathbb{K}^{\sum_{r=1}^{k} m_{r} n_{r} s \times \sum_{r=1}^{k} m_{r} n_{r} s}$ is a block $\sum_{r=1}^{k} m_{r} \times \sum_{r=1}^{k} m_{r}$ matrix with blocks

$$
(A \odot Z)_{i j}^{\left(r r^{\prime}\right)}=\sum_{\kappa=1}^{\ell_{r^{\prime}}} A_{i \kappa}^{\left(r r^{\prime}\right)} \otimes Z_{\kappa j}^{\left(r^{\prime}\right)} \in \mathbb{K}^{n_{r} \times n_{r^{\prime}}} \otimes \mathbb{K}^{s \times s} \cong \mathbb{K}^{n_{r} s \times n_{r^{\prime}} s},
$$

$i=1, \ldots, m_{r}, j=1, \ldots, m_{r^{\prime}}$.

Proof. It is clear that the inclusion " $\supseteq$ " holds in both the equalities in (2.4).

Conversely, let $Z \in \operatorname{edom}_{s} \mathfrak{R}$ for some $s \in \mathbb{N}$. We will show that $\operatorname{det}(I-A \odot Z) \neq 0$. Let

$$
\mathcal{L}=\left(\mathcal{L}_{i j}^{(r)}\right)_{r=1, \ldots, k, i=1, \ldots, \ell_{r}, j=1, \ldots, m_{r}}, \quad \mathcal{R}=\left(\mathcal{R}_{i j}^{(r)}\right)_{i=1, \ldots, \ell_{r}, j=1, \ldots, m_{r}}
$$

\footnotetext{
${ }^{2}$ Here, similarly to the convention we made in a footnote on the front page of the paper, we assume that a matrix block is void if the number of its rows/columns is 0 .
} 
be the $d$-tuples of left and right backward shifts, where $d=\sum_{r=1}^{k} \ell_{r} m_{r}$. For a word $w=$ $g_{i_{0} j_{0}}^{\left(r_{0}\right)} \cdots g_{i_{N} j_{N}}^{\left(r_{N}\right)} \in \mathcal{G}_{d}$, we set $\mathcal{L}^{w}=\mathcal{L}_{i_{0} j_{0}}^{\left(r_{0}\right)} \cdots \mathcal{L}_{i_{N} j_{N}}^{\left(r_{N}\right)}, \mathcal{R}^{w}=\mathcal{R}_{i_{0} j_{0}}^{\left(r_{0}\right)} \cdots \mathcal{R}_{i_{N} j_{N}}^{\left(r_{N}\right)}$. Then for any $w=$ $g_{i_{0} j_{0}}^{\left(r_{0}\right)} \cdots g_{i_{N} j_{N}}^{\left(r_{N}\right)} \in \mathcal{G}_{d}$ with $N \geq 1$ we obtain

$$
\begin{aligned}
\mathcal{R}^{w}(R)= & \mathcal{R}^{w}\left(C z_{n}\left(I-A z_{n}\right)^{-1} B\right) \\
& =\mathcal{R}^{w}\left(C\left(I-z_{n} A\right)^{-1} z_{n} B\right)=C\left(I-z_{n} A\right)^{-1}\left(\mathbf{e}_{i_{0}}^{\left(r_{0}\right)} \otimes A_{j_{0} i_{1}}^{\left(r_{0} r_{1}\right)} \cdots A_{j_{N-1} i_{N}}^{\left(r_{N} r_{N}\right)} B_{j_{N}}^{\left(r_{N}\right)}\right),
\end{aligned}
$$

where $\mathbf{e}_{i_{0}}^{\left(r_{0}\right)}$ is the $\left(\sum_{r=1}^{r_{0}-1} \ell_{r}+i_{0}\right)$-th standard basis vector of $\mathbb{K}^{\sum_{r=1}^{k} \ell_{r}}$. Since $Z \in \operatorname{edom}_{s} \mathfrak{R}$, we have $Z \in \operatorname{edom}_{s} \mathcal{R}^{w} \mathfrak{R}=\operatorname{edom}_{s} \mathcal{R}^{w}(R)$. Therefore, the $\alpha s \times \beta s$ matrix-valued rational function

$$
\left(C \otimes I_{s}\right)\left(I-X \odot_{\mathrm{op}} A\right)^{-1}\left(\mathbf{e}_{i_{0}}^{\left(r_{0}\right)} \otimes A_{j_{0} i_{1}}^{\left(r_{0} r_{1}\right)} \cdots A_{j_{N-1} i_{N}}^{\left(r_{N-1} r_{N}\right)} B_{j_{N}}^{\left(r_{N}\right)} \otimes I_{s}\right)
$$

in the commuting variables $\left(X_{i j}^{(r)}\right)_{\mu \nu}, r=1, \ldots, k, i=1, \ldots, \ell_{r}, j=1, \ldots, m_{r}, \mu, \nu=$ $1, \ldots, s$, is regular at $X=Z$, where $X \odot_{\text {op }} A \in \mathbb{K}^{\sum_{r=1}^{k} \ell_{r} n_{r} s \times \sum_{r=1}^{k} \ell_{r} n_{r} s}$ is a block $\sum_{r=1}^{k} \ell_{r} \times$ $\sum_{r=1}^{k} \ell_{r}$ matrix with blocks

$$
\left(X \odot_{\mathrm{op}} A\right)_{i j}^{\left(r r^{\prime}\right)}=\sum_{\kappa=1}^{m_{r}} A_{\kappa j}^{\left(r r^{\prime}\right)} \otimes X_{i \kappa}^{(r)} \in \mathbb{K}^{n_{r} \times n_{r^{\prime}}} \otimes \mathbb{K}^{s \times s} \cong \mathbb{K}^{n_{r} s \times n_{r^{\prime}} s} .
$$

The controllability assumption implies that the $\alpha s \times \sum_{r=1}^{k} \ell_{r} n_{r} s$ matrix-valued rational function $\left(C \otimes I_{s}\right)\left(I-X \odot_{\mathrm{op}} A\right)^{-1}$ is regular at $X=Z$. Therefore, the $\alpha s \times \sum_{r=1}^{k} m_{r} n_{r} s$ matrixvalued rational function

$$
\left(C \otimes I_{s}\right)\left(I-X \odot_{\text {op }} A\right)^{-1}\left(X \odot_{\text {op }} I_{\sum_{r=1}^{k} m_{r} n_{r}}\right)=(C \odot X)(I-A \odot X)^{-1}
$$

is regular at $X=Z$. In other words, $Z \in \operatorname{edom}_{s} R^{\prime}$ where

$$
R^{\prime}=C z_{n}\left(I-A z_{n}\right)^{-1}
$$

is an $\alpha \times \sum_{r=1}^{k} m_{r} n_{r}$ matrix-valued noncommutative rational expression.

Next, for any $w=g_{i_{0} j_{0}}^{\left(r_{0}\right)} \cdots g_{i_{N} j_{N}}^{\left(r_{n}\right)} \in \mathcal{G}_{d}$ with $N \geq 1$ we set $w^{\top}=g_{i_{N} j_{N}}^{\left(r_{N}\right)} \cdots g_{i_{0} j_{0}}^{\left(r_{0}\right)}$. Then we have

$$
\mathcal{L}^{w^{\top}}\left(R^{\prime}\right)=\left(\left(\mathbf{f}_{j_{0}}^{\left(r_{0}\right)}\right)^{\top} \otimes C_{i_{N}}^{\left(r_{N}\right)} A_{j_{N} i_{N-1}}^{\left(r_{N} r_{N-1}\right)} \cdots A_{j_{1} i_{0}}^{\left(r_{1} r_{0}\right)}\right)\left(I-A z_{n}\right)^{-1}
$$

where $\mathbf{f}_{j_{0}}^{\left(r_{0}\right)}$ is the $\left(\sum_{r=1}^{r_{0}-1} m_{r}+j_{0}\right)$-th standard basis vector of $\mathbb{K}^{\sum_{r=1}^{k} m_{r}}$. Since $Z \in \operatorname{edom}_{s} R^{\prime}$, we have $Z \in \operatorname{edom}_{s} \mathcal{L}^{w^{\top}}\left(R^{\prime}\right)$. Therefore, the $\alpha s \times \sum_{r=1}^{k} m_{r} n_{r} s$ matrix-valued rational function

$$
\left(\left(\mathbf{f}_{j_{0}}^{\left(r_{0}\right)}\right)^{\top} \otimes C_{i_{N}}^{\left(r_{N}\right)} A_{j_{N} i_{N-1}}^{\left(r_{N} r_{N-1}\right)} \cdots A_{j_{1} i_{0}}^{\left(r_{1} r_{0}\right)} \otimes I_{s}\right)(I-A \odot X)^{-1}
$$

in the commuting variables $\left(X_{i j}^{(r)}\right)_{\mu \nu}, r=1, \ldots, k, i=1, \ldots, \ell_{r}, j=1, \ldots, m_{r}, \mu, \nu=$ $1, \ldots, s$, is regular at $X=Z$. The observability assumption implies that the $\sum_{r=1}^{k} m_{r} n_{r} s \times$ $\sum_{r=1}^{k} m_{r} n_{r} s$ matrix-valued rational function

$$
(I-A \odot X)^{-1}
$$

is regular at $X=Z$. Then so is the rational function

$$
\operatorname{det}(I-A \odot X)^{-1}=(\operatorname{det}(I-A \odot X))^{-1},
$$


i.e., $\operatorname{det}(I-A \odot Z) \neq 0$, as required.

Corollary 2.2. The variety of singularities of an $\alpha \times \beta$ matrix-valued rational function $f$ which can be represented as a restriction $R_{1}$ of an $\alpha \times \beta$ matrix-valued noncommutative rational expression $R$ of the form (2.1) satisfying the assumptions of Theorem 2.1 (i.e., which is obtained from $R$ by replacing the noncommuting indeterminates $z_{i j}^{(r)}$ by the commuting ones), is given by

$$
\left\{Z=\left(Z^{(1)}, \ldots, Z^{(k)}\right) \in \mathbb{K}^{\ell_{1} \times m_{1}} \times \cdots \times \mathbb{K}^{\ell_{k} \times m_{k}}: \operatorname{det}\left(I-A Z_{n}\right)=0\right\},
$$

where $Z_{n}=\bigoplus_{r=1}^{k}\left(Z^{(r)} \otimes I_{n_{r}}\right)$.

Proof. Clearly, the variety of singularities of $f$ coincides with

$$
\left(\mathbb{K}^{\ell_{1} \times m_{1}} \times \cdots \times \mathbb{K}^{\ell_{k} \times m_{k}}\right) \backslash \operatorname{edom}_{1} R
$$

The result then follows from Theorem 2.1.

We will also need to make use of the inverse of a noncommutative rational function, and of the fact that the minimality of a realization carries over to the corresponding realization of the inverse. We recall from [5, Section 4] that if $\mathfrak{R}$ is an $\alpha \times \alpha$ matrix-valued noncommutative rational function over a field $\mathbb{K}$ represented by the noncommutative rational expression (2.1) with $D$ invertible, then its inverse exists and has a realization

$$
R^{-1}=D^{\times}+C^{\times} z_{n}\left(I-A^{\times} z_{n}\right)^{-1} B^{\times},
$$

where

$$
\left[\begin{array}{ll}
A^{\times} & B^{\times} \\
C^{\times} & D^{\times}
\end{array}\right]=\left[\begin{array}{cc}
A-B D^{-1} C & B D^{-1} \\
-D^{-1} C & D^{-1}
\end{array}\right]
$$

Proposition 2.3. Assume that the realization of $R$ in (2.1) is minimal and that $D$ is invertible. Then the realization of $R^{-1}$ given via (2.5) and (2.6) is also minimal.

Proof. It suffices to verify the controllability and observability for the realization of $R^{-1}$. Notice that the blocks of $A^{\times}$and $B^{\times}$are $A_{j i}^{\times\left(r r^{\prime}\right)}=A_{j i}^{\left(r r^{\prime}\right)}-B_{j}^{(r)} D^{-1} C_{i}^{\left(r^{\prime}\right)}$ and $B_{j}^{\times(r)}=B_{j}^{(r)} D^{-1}$, respectively. Thus, for the controllability, we need to check that for each $r_{0} \in\{1, \ldots, N\}$ and $j_{0} \in\left\{1, \ldots, m_{r_{0}}\right\}$ one has

$$
\sum_{N \in \mathbb{N}, \gamma \in\{1, \ldots, N\}, i_{\gamma} \in\left\{1, \ldots, \ell_{r_{\gamma}}\right\}, j_{\gamma} \in\left\{1, \ldots, m_{r_{\gamma}}\right\}} \operatorname{range}\left(A_{j_{0} i_{1}}^{\times\left(r_{0} r_{1}\right)} \cdots A_{j_{N-1} i_{N}}^{\times\left(r_{N-1} r_{N}\right)} B_{j_{N}}^{\times\left(r_{N}\right)}\right)=\mathbb{K}^{n_{r_{0}}} .
$$

Clearly, range $B_{j}^{\times(r)}=\operatorname{range} B_{j}^{(r)} D^{-1}=\operatorname{range} B_{j}^{(r)}$ for all $r=1, \ldots, k$ and $j=1, \ldots, m_{r}$. Next,

$$
\begin{aligned}
& \operatorname{range}\left(A_{j_{0} i_{1}}^{\times\left(r_{0} r_{1}\right)} B_{j_{1}}^{\times\left(r_{1}\right)}\right)+\operatorname{range} B_{j_{0}}^{\times\left(r_{0}\right)} \\
&=\operatorname{range}\left(\left(A_{j_{0} i_{1}}^{\left(r_{0} r_{1}\right)}-B_{j_{0}}^{\left(r_{0}\right)} D^{-1} C_{i_{1}}^{\left(r_{1}\right)}\right) B_{j_{1}}^{\left(r_{1}\right)} D^{-1}\right)+\operatorname{range} B_{j_{0}}^{\left(r_{0}\right)} D^{-1} \\
&=\operatorname{range}\left(A_{j_{0} i_{1}}^{\left(r_{0} r_{1}\right)} B_{j_{1}}^{\left(r_{1}\right)}\right)+\operatorname{range} B_{j_{0}}^{\left(r_{0}\right)} .
\end{aligned}
$$

Continuing this way one sees that the left hand sides of (2.2) and (2.7) are the same, and thus (2.7) follows from (2.2). In a similar way, one shows the observability. 


\section{Contractive Determinantal Depresentations of Stable polynomials on a MATRIX POLYBALL}

The main result of the paper is the following.

Theorem 3.1. Let $p$ be an irreducible polynomial in the commuting indeterminates $z_{i j}^{(r)}$, $r=1, \ldots, k, i=1, \ldots, \ell_{r}, j=1, \ldots, m_{r}$, with $p(0)=1$, which is strongly stable with respect to the matrix polyball $\mathbb{B}^{\ell_{1} \times m_{1}} \times \cdots \times \mathbb{B}^{\ell_{k} \times m_{k}}$. Then there exist $n=\left(n_{1}, \ldots, n_{k}\right) \in \mathbb{Z}_{+}^{k}$ and $a$ strict contraction $K \in \mathbb{C}^{\sum_{r=1}^{k} m_{r} n_{r} \times \sum_{r=1}^{k} \ell_{r} n_{r}}$ so that

$$
p=\operatorname{det}\left(I-K Z_{n}\right),
$$

where $Z_{n}=\bigoplus_{r=1}^{k}\left(Z^{(r)} \otimes I_{n_{r}}\right)$ and $Z^{(r)}=\left[z_{i j}^{(r)}\right] \in \mathbb{C}^{\ell_{r} \times m_{r}}$.

Proof. Since $p$ has no zeros in the closed unit polyball $\overline{\mathbb{B}}^{\ell_{1} \times m_{1}} \times \cdots \times \overline{\mathbb{B}}^{\ell_{k} \times m_{k}}$, we have that $p$ has no zeros in $\rho \overline{\mathbb{B}}^{\ell_{1} \times m_{1}} \times \cdots \times \rho \overline{\mathbb{B}}^{\ell \times m_{k}}$ for some $\rho>1$ sufficiently close to 1 . Thus the rational function $g=1 / p$ is regular on $\rho \overline{\mathbb{B}}^{\ell_{1} \times m_{1}} \times \cdots \times \rho \overline{\mathbb{B}}^{\ell_{k} \times m_{k}}$, and the rational function $g_{\rho}$ defined by $g_{\rho}(z)=g(\rho z)$ is regular on $\overline{\mathbb{B}}^{\ell_{1} \times m_{1}} \times \cdots \times \overline{\mathbb{B}}^{\ell_{k} \times m_{k}}$. By [11, Lemma 3.3], $\left\|g_{\rho}\right\|_{\mathcal{A}, Z}<\infty$, where the corresponding Agler norm $\|\cdot\|_{\mathcal{A}, Z}=\|\cdot\|_{\mathcal{A}, P}$ is defined as in (1.5) with $\mathbf{P}=Z=\bigoplus_{r=1}^{k} Z^{(r)}$. Thus we can find a constant $c>0$ so that $\left\|c g_{\rho}\right\|_{\mathcal{A}, Z}<1$. By [11, Theorem 3.4] applied to $F=c g_{\rho}$, we obtain a $n=\left(n_{1}, \ldots, n_{k}\right) \in \mathbb{Z}_{+}^{k}$ and a contractive colligation matrix $\left[\begin{array}{cc}A & B \\ C\end{array}\right]$ of size $\left(\sum_{r=1}^{k} m_{r} n_{r}+1\right) \times\left(\sum_{r=1}^{k} \ell_{r} n_{r}+1\right)$ such that

$$
c g_{\rho}=D+C Z_{n}\left(I-A Z_{n}\right)^{-1} B, \quad Z_{n}=\bigoplus_{r=1}^{k}\left(Z^{(r)} \otimes I_{n_{r}}\right) .
$$

Therefore

$$
c g=D+C\left(\rho^{-1} Z_{n}\right)\left(I-A\left(\rho^{-1} Z_{n}\right)\right)^{-1} B=D+\rho^{-1} C Z_{n}\left(I-\rho^{-1} A Z_{n}\right)^{-1} B .
$$

Then we lift the rational function $c g$ to a noncommutative rational expression using the same realization formula,

$$
\left.D+C^{\prime} z_{n}\left(I-A^{\prime} z_{n}\right)\right)^{-1} B
$$

now with $z_{n}=\bigoplus_{r=1}^{k}\left(z^{(r)} \otimes I_{n_{r}}\right)$ and the entries $z_{i j}^{(r)}$ of matrices $z^{(r)}$ being noncommuting indeterminates, $r=1, \ldots, k, i=1, \ldots, \ell_{r}, j=1, \ldots, m_{r}$, and $A^{\prime}=\rho^{-1} A, C^{\prime}=\rho^{-1} C$ (cf. (2.1)). Notice that the colligation matrix $\left[\begin{array}{cc}A^{\prime} & B \\ C^{\prime} & D\end{array}\right]$ is contractive, with $\left\|A^{\prime}\right\|<1$ and $\left\|C^{\prime}\right\|<1$. Compressing the underlying noncommutative structured system to a minimal one (see [5, Theorem 7.1]), we obtain a noncommutative rational expression

$$
R=D_{\min }+C_{\min } z_{n_{\min }}\left(I-A_{\min } z_{n_{\min }}\right)^{-1} B_{\min }
$$

whose colligation matrix $\left[\begin{array}{cc}A_{\min } & B_{\min } \\ C_{\min } & D_{\min }\end{array}\right]$ is still contractive and such that $\left\|A_{\min }\right\|<1$ and $\left\|C_{\text {min }}\right\|<1$. By Proposition [2.3, we also obtain a minimal noncommutative structured system realization of $R^{-1}$,

$$
R^{-1}=D_{\min }^{\times}+C_{\min }^{\times} z_{n_{\min }}\left(I-A_{\min }^{\times} z_{n_{\min }}\right)^{-1} B_{\min }^{\times},
$$


with the colligation matrix

$$
\left[\begin{array}{cc}
A_{\min }^{\times} & B_{\min }^{\times} \\
C_{\min }^{\times} & D_{\min }^{\times}
\end{array}\right]=\left[\begin{array}{cc}
A_{\min }-B_{\min } D_{\min }^{-1} C_{\min } & B_{\min } D_{\min }^{-1} \\
-D_{\min }^{-1} C_{\min } & D_{\min }^{-1}
\end{array}\right]
$$

(cf., (2.5)-(2.6) ). Applying Theorem 2.1 to $R^{-1}$ and Corollary 2.2 to $R_{1}^{-1}=p / c$, we obtain that the singularity set of the polynomial $p / c$ (which is the empty set), agrees with

$$
\left\{Z=\left(Z^{(1)}, \ldots, Z^{(k)}\right) \in \mathbb{C}^{\ell_{r_{1}} \times m_{r_{1}}} \times \cdots \times \mathbb{C}^{\ell_{r_{k}} \times m_{r_{k}}}: \operatorname{det}\left(I-A_{\min }^{\times} Z_{n_{\min }}\right)=0\right\},
$$

which is possible only if $\operatorname{det}\left(I-A_{\min }^{\times} Z_{n_{\min }}\right) \equiv 1$.

Next, from the following two factorizations,

$$
\begin{aligned}
& {\left[\begin{array}{cc}
I-A_{\min } Z_{n_{\min }} & B_{\min } \\
-C_{\min } Z_{n_{\min }} & D_{\min }
\end{array}\right]} \\
& =\left[\begin{array}{cc}
I & 0 \\
-C_{\min } Z_{n_{\min }}\left(I-A_{\min } Z_{n_{\min }}\right)^{-1} & I
\end{array}\right]\left[\begin{array}{cc}
I-A_{\min } Z_{n_{\min }} & 0 \\
0 & c / p
\end{array}\right]\left[\begin{array}{cc}
I & \left(I-A_{\min } Z_{n_{\min }}\right)^{-1} B \\
0 & I
\end{array}\right] \\
& =\left[\begin{array}{cc}
I & B_{\min }^{\times} \\
0 & I
\end{array}\right]\left[\begin{array}{cc}
I-A_{\min }^{\times} Z_{n_{\min }} & 0 \\
0 & D_{\min }
\end{array}\right]\left[\begin{array}{cc}
I & 0 \\
C_{\min }^{\times} Z_{n_{\min }} & I
\end{array}\right],
\end{aligned}
$$

we obtain that

$$
\begin{array}{r}
\operatorname{det}\left[\begin{array}{cc}
I-A_{\min } Z_{n_{\min }} & B_{\min } \\
-C_{\min } Z_{n_{\min }} & D_{\min }
\end{array}\right]=\frac{c}{p} \operatorname{det}\left(I-A_{\min } Z_{n_{\min }}\right)=D_{\min } \operatorname{det}\left(I-A_{\min }^{\times} Z_{n_{\min }}\right) \\
=D_{\min }=\frac{c}{p(0)}=c .
\end{array}
$$

Therefore, $p=\operatorname{det}\left(I-A_{\min } Z_{n_{\min }}\right)$. Since $A_{\min }$ is a strict contraction, we obtain that (3.1) is true with $K=A_{\min }$ and $n_{\min }$ in the place of $n$.

Corollary 3.2. Every strongly $\mathbb{D}^{d}$-stable polynomial $p$ is an eventual Agler denominator, i.e., there exists $n=\left(n_{1}, \ldots, n_{d}\right) \in \mathbb{Z}_{+}^{d}$ such that the rational inner function

$$
\frac{z^{n} \bar{p}(1 / z)}{p(z)}
$$

is in the Schur-Agler class. Here for $z=\left(z_{1}, \ldots, z_{d}\right)$ we set $1 / z=\left(1 / z_{1}, \ldots, 1 / z_{d}\right), \bar{p}(z)=$ $\overline{p\left(\bar{z}_{1}, \ldots, \bar{z}_{d}\right)}$, and $z^{n}=z_{1}^{n_{1}} \cdots z_{d}^{n_{d}}$.

Proof. By Theorem 3.1 applied to the polydisk case, $p$ has a strictly contractive determinantal representation (3.1). By [12, Theorem 5.2], $p$ is an eventual Agler denominator. Moreover, $n$ in (3.2) can be chosen the same as in a (not necessarily strictly) contractive determinantal representation (3.1) for $p$.

\section{REFERENCES}

[1] J. Agler. On the representation of certain holomorphic functions defined on a polydisc, In Topics in operator theory: Ernst D. Hellinger Memorial Volume, Oper. Theory Adv. Appl., Vol. 48, pp. 47-66, Birkhäuser, Basel, 1990.

[2] C.-G. Ambrozie and D. A. Timotin. Von Neumann type inequality for certain domains in $\mathbb{C}^{n}$. Proc. Amer. Math. Soc. 131 (2003), no. 3, 859-869 (electronic).

[3] D. Z. Arov. Passive linear steady-state dynamical systems. (Russian) Sibirsk. Mat. Zh. 20 (1979), no. $2,211-228,457$. 
[4] J. A. Ball and V. Bolotnikov. Realization and interpolation for Schur-Agler-class functions on domains with matrix polynomial defining function in $\mathbb{C}^{n}$. J. Funct. Anal. 213 (2004), no. 1, 45-87.

[5] J. A. Ball, G. Groenewald, and T. Malakorn. Structured noncommutative multidimensional linear systems. SIAM J. Control Optim. 44 (2005), no. 4, 1474-1528.

[6] J. A. Ball and D. S. Kaliuzhnyi-Verbovetskyi. Rational Cayley inner Herglotz-Agler functions: Positivekernel decompositions and transfer-function realizations. Linear Algebra Appl. 456 (2014), 138-156.

[7] S. Basu and A. Fettweis. New results on stable multidimensional polynomials. II. Discrete case. IEEE Trans. Circuits and Systems 34 (1987), 1264-1274.

[8] J. Borcea, P. Brändén, and T. M. Liggett. Negative dependence and the geometry of polynomials. $J$. Amer. Math. Soc. 22 (2009), no. 2, 521-567.

[9] J. C. Doyle. Analysis of feedback systems with structured uncertainties. Proc. IEE-D 129 (1982), no. 6, 242-250.

[10] A. Grinshpan, D. S. Kaliuzhnyi-Verbovetskyi, V. Vinnikov, and H. J. Woerdeman. Stable and real-zero polynomials in two variables. Multidim. Syst. Sign. Process. Published online.

[11] A. Grinshpan, D. S. Kaliuzhnyi-Verbovetskyi, V. Vinnikov, and H. J. Woerdeman. Matrix-valued Hermitian Positivstellensatz, lurking contractions, and contractive determinantal representations of stable polynomials. Preprint, arXiv 1501.05527.

[12] A. Grinshpan, D. S. Kaliuzhnyi-Verbovetskyi, and H. J. Woerdeman. Norm-constrained determinantal representations of multivariable polynomials. Complex Anal. Oper. Theory 7 (2013), 635-654.

[13] A. Grinshpan, D. S. Kaliuzhnyi-Verbovetskyi, and H. J. Woerdeman. The Schwarz lemma and the SchurAgler class. Proceedings of the 21st International Symposium on Mathematical Theory of Networks and Systems, 2014.

[14] L. Gurvits. Van der Waerden/Schrijver-Valiant like conjectures and stable (aka hyperbolic) homogeneous polynomials: one theorem for all. With a corrigendum. Electron. J. Combin., 15 (2008), no. 1, Research Paper 66, 26 pp.

[15] D. S. Kaliuzhnyi-Verbovetskyi and V. Vinnikov, Foundations of Free Non-commutative Function Theory. Math Surveys and Monographs, Vol. 199, AMS, 2014, 183 pp.

[16] D. S. Kaliuzhnyi-Verbovetskyi and V. Vinnikov, Noncommutative rational functions, their differencedifferential calculus and realizations. Multidimens. Syst. Signal Process. 23 (2012), no. 1-2, 49-77.

[17] D. S. Kaliuzhnyi-Verbovetskyi and V. Vinnikov. Singularities of rational functions and minimal factorizations: The noncommutative and the commutative setting. Linear Algebra Appl. 430 (2009), no. 4, 869-889.

[18] G. Knese. Rational inner functions in the Schur-Agler class of the polydisk. Publ. Mat., 55 (2011), 343-357.

[19] A. Kummert. 2-D stable polynomials with parameter-dependent coefficients: generalizations and new results. IEEE Trans. Circuits Systems I: Fund. Theory Appl. 49 (2002), 725-731.

[20] A. Kummert. Synthesis of two-dimmensional lossless $m$-ports with prescribed scattering matrix. Circuits Systems Signal Processing 8 (1989), no. 1, 97-119.

[21] A. Kummert. A parametric representation for $k$-variable Schur polynomials. IEEE Trans. Circuits Systems 37 (1990), no. 10, 1288-1291.

[22] L. Li , L. Xu, and Z. Lin. Stability and stabilisation of linear multidimensional discrete systems in the frequency domain. Int. J. Control 86 (2013), no. 11, 1969-1989.

[23] M. Scheicher. Robustly stable multivariate polynomials. Multidimens. Syst. Signal Process. 24 (2013), no. $1,23-50$.

[24] J. L. Taylor. A joint spectrum for several commuting operators. J. Functional Analysis 6 (1970), 172191.

[25] J. L. Taylor. The analytic-functional calculus for several commuting operators. Acta Math. 125 (1970), $1-38$.

[26] D. G. Wagner. Multivariate stable polynomials: theory and applications. Bull. Amer. Math. Soc. 48 (2011), no. 1, 53-84. 
Department of Mathematics, Drexel University, 3141 Chestnut St., Philadelphia, Pa, 19104

E-mail address: \{tolya,dmitryk, hugo\}@math.drexel.edu

Department of Mathematics, Ben-Gurion University of the Negev, Beer-Sheva, Israel, 84105

E-mail address: vinnikov@math.bgu.ac.il 